\title{
La promotion de l'animal au niveau de l'humain? La reconnaissance de la personnalité animale, nouveau credo des juristes
}

\section{Séverine Nadaud}

\section{CpenEdition}

\section{Journals}

Édition électronique

URL : https://journals.openedition.org/rdr/1708

DOI : $10.4000 /$ rdr. 1708

ISSN : 2534-7462

Éditeur

Presses universitaires de Strasbourg

\section{Édition imprimée}

Date de publication : 2 décembre 2021

Pagination : 101-112

ISBN : 979-10-344-0097-3

ISSN : 2493-8637

\section{Référence électronique}

Séverine Nadaud, « La promotion de l'animal au niveau de l'humain ? La reconnaissance de la personnalité animale, nouveau credo des juristes », Revue du droit des religions [En ligne], 12 | 2021, mis en ligne le 02 décembre 2021, consulté le 02 mai 2022. URL : http://journals.openedition.org/rdr/1708 ; DOl : https://doi.org/10.4000/rdr.1708

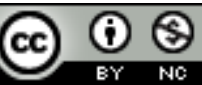

La revue du droit des religions est mise à disposition selon les termes de la Creative Commons Attribution - Pas d'Utilisation Commerciale 4.0 International - CC BY-NC 4.0. 


\section{La promotion de l'animal au niveau de l'humain? La reconnaissance de la personnalité animale, nouveau credo des juristes}

\section{Séverine NADAUD}

Université de Limoges, Observatoire des mutations institutionnelles et juridiques (OMIJ)

\section{RÉSUMÉ}

Depuis quelques années, des juristes prônent la reconnaissance d'une personnalité juridique technique qui permettrait de faire bénéficier les animaux de droits spécifiques et adaptés. Loin de l'idée de promouvoir l'animal au niveau de l'être humain, cette personnalité animale pourrait être reconnue par le juge. Elle constituerait un progrès pour la condition animale et ne conduirait pas à minorer les droits de l'homme mais viendrait au contraire les conforter et les enrichir.

\section{Abstract}

For a few years now, some legal experts are in favour of providing legal personality to animals so that they could benefit from specific and adapted rights. Far from promoting the animal to the level of the human being, this legal personality could be recognized by the judge. It would constitute progress for the animal condition and would not lead to a weakening of the rights of human beings. On the contrary, it would reinforce and enhance human rights. 
$\mathrm{O}$ n peut considérer avec Mireille Delmas-Marty que « tout se passe comme si le droit était devenu ou supposé devenir le substitut des religions et des idéologies ${ }^{1} »$, le droit se révélant souvent un formidable instrument pour faire avancer certaines questions sociétales, certaines causes. Ainsi s'est constituée une communauté de juristes qui prophétise depuis quelques décennies déjà que rien ne pourra empêcher demain l'attribution de la personnalité juridique à de nouvelles entités, au premier rang desquelles figurent les animaux ${ }^{2}$.

Notre droit n'a eu de cesse de connaître de notables évolutions en faveur d'une plus grande protection de l'animal au point que s'est progressivement édifiée une nouvelle branche du droit, un «droit de l'animal ${ }^{3} »$ pour certains, un «droit animalier ${ }^{4} »$ pour d'autres. Les avancées se sont tout d'abord concrétisées sur le plan pénal ${ }^{5}$. La loi Grammont du 2 juillet 1850 , préliminaire à l'idée d'une protection animale, disposait que «seront punis d'une amende de cinq à quinze francs, et pourront l'être d'un à cinq jours de prison, ceux qui auront exercé publiquement et abusivement des mauvais traitements envers les animaux domestiques». Il faudra toutefois attendre le décret Michelet du 7 septembre 1959 pour voir disparaître l'exigence de publicité pour l'infraction de «mauvais traitement» et prévoir la remise de l'animal maltraité à une œuvre de protection. Si la loi du 19 novembre 1963 a pour sa part permis de réprimer les actes de cruauté sans exiger là non plus qu'ils aient été exercés en public, le nouveau Code pénal de 1992 marquera une étape importante en ne faisant plus figurer les infractions contre les animaux dans le même chapitre que celui réservé aux infractions contre

1. M. Delmas-Marty, «Avant-propos», in Pour un droit commun, Paris, Seuil, 1994.

2. «[...] depuis quelques années, d'autres entités que les êtres humains sont de plus en plus sérieusement candidates à les rejoindre dans le monde bariolé des personnes juridiques: l'Humanité $[\ldots]$; les robots [...] et quelques éléments de la nature [...]. La candidature la plus avancée est, à n'en plus douter, celle des animaux [...]»: J.-P. MARGUÉNAUD, F. Burgat et J. Leroy, «La personnalité animale», D. 2020, p. 28. V. aussi les actes du colloque «Droits des êtres humains et droits des autres entités: une nouvelle frontière? ", organisé par l'université de Montpellier les 16 et 17 mai 2019, à paraître aux éditions Mare \& Martin sous la dir. de C. Vial et J.-P. Marguénaud.

3. S. Antoine, «Le droit de l'animal: évolution et perspectives», D. 1996, p. 126; Le droit de l'animal, Paris, Légis-France, 2007; A.-C. Lomellini-Dereclenne et C. Mercier, Le droit de l'animal, Paris, LGDJ, 2017; O. Le Bot, Introduction au droit de l'animal, [s.l.], Le Bot, 2018; F. Dossche (dir.), Le droit des animaux. Perspectives d'avenir, Bruxelles, Larcier, 2019.

4. J.-P. Marguénaud, F. Burgat et J. Leroy, Le droit animalier, Paris, PUF, 2016; J.-P. MarGuÉNAud et X. Perrot, «Le droit animalier, de l'anecdotique au fondamental», D. 2017, p. 996; M. FAlaise, Droit animalier, Levallois-Perret, Bréal, 2018.

5. V. par ex. les évolutions pénales retracées par S. ANTOINE, «Le droit de l'animal: évolution et perspectives», art. cit. 
les biens ${ }^{6}$. Dans un second temps, dépassant le cadre pénal, la loi sur la protection de la nature du 10 juillet $1976^{7}$ reconnaît pour la première fois la sensibilité à l'animal proche de l'homme et s'attache à s'intéresser à l'animal lui-même, à ses impératifs biologiques, à ses besoins, à son bien-être ${ }^{8}$. Ce texte sera le point de départ d'une abondante législation ${ }^{9}$ principalement intégrée dans divers codes (Code rural et de la pêche maritime, Code civil, etc.) afin de protéger l'animal notamment en encadrant le droit de propriété de l'homme par des textes influencés par les avancées imposées par les droits européens ${ }^{10}$. On assiste dès lors à une prolifération de dispositions à dominance technique destinées à améliorer les conditions de vie des animaux domestiques, plus particulièrement ceux qui sont les plus asservis aux besoins de l'homme. Mais la plus grande avancée juridique se fera grâce à l'amendement Glavany ${ }^{11}$ modifiant l'article 2 de la loi n ${ }^{\circ} 2015$ 177 du 16 février 2015 et qui aboutira à la création de l'article 515-14 du Code civil en vertu duquel «Les animaux sont des êtres vivants doués de sensibilité. Sous réserve des lois qui les protègent, les animaux sont soumis au régime des biens».

6. J.-P. Marguénaud, «L'animal dans le nouveau code pénal», D. 1995, chron. p. 187; M. DANTI-JUAN, «La contribution du nouveau code pénal au débat sur la nature juridique de l'animal », RD rur. 1996, p. 477.

7. En tant que grande loi environnementale, la loi sur la protection de la nature du 10 juillet 1976 a également affirmé pour la première fois que la protection des espèces sauvages et des espaces dans lesquels elles évoluent est d'intérêt général. Elle sera donc elle aussi le point de départ de l'édiction de dispositions toujours plus nombreuses destinées à protéger les espèces les plus menacées.

8. S. BRELS, Le droit du bien-être animal dans le monde: évolution et universalisation, Paris, L'Harmattan, 2017; S. Hild et L. SChweitzer (dir.), Le bien-être animal: de la science au droit, Paris, L'Harmattan, 2018.

9. À noter toutefois que ces avancées ne concerneront principalement que l'animal domestique. En effet, si la loi pour la reconquête de la biodiversité du 8 août 2016 a permis de remplacer les mots «espèces animales» par les mots «êtres vivants » à l'article L. 110-1 du Code de l'environnement et donc d'opérer un début de singularisation des animaux principalement appréhendés par le biais de l'espèce, elle n'a pas malheureusement conduit à reconnaître leur sensibilité à l'égal de ce qu'avait fait l'amendement Glavany. V. sur ce point notre contribution lors de l'université d'été organisée en juillet 2019 par la faculté de droit et sciences sociales de Poitiers: "Les apports de la loi pour la reconquête de la biodiversité à la protection des animaux sauvages», in M. FAURE-ABBAD et D. Gantschnig (dir.), Les animaux, Poitiers, Presses universitaires juridiques de Poitiers, 2020, p. 223.

10. O. Dubos et J.-P. MarguÉnaud, Les animaux et les droits européens. Au-delà de la distinction entre les hommes et les choses, Paris, Pédone, 2009.

11. J.-P. MARguÉNAUd, «La modernisation des dispositions du code civil relatives aux animaux: l'échappée belle. Commentaire de l'article 2 de la loi n² 2015-177 du 16 février 2015», RJE, $\mathrm{n}^{\circ} 2,2015$, p. 257-263. 
Mais si ce droit de l'animal ou animalier en tant que droit objectif s'est développé et continue à se développer, certains juristes souhaiteraient toutefois passer à une nouvelle étape, qui serait celle de l'attribution de droits subjectifs à l'animal. Cette volonté s'appuie sur la constatation que ce corpus étoffé de droit objectif reste encore très perfectible: s'il est davantage protecteur de la bien-traitance de l'animal proche de l'homme (c'est-à-dire de l'animal domestique ou de l'animal sauvage seulement s'il est apprivoisé, détenu ou captif), ce droit n'offre toutefois qu'une protection nuancée du fait de l'admission de certaines dérogations soucieuses de préserver notamment « des rites religieux, des traditions culturelles et des patrimoines régionaux » comme le rappelle l'article 13 du Traité sur le fonctionnement de l'Union européenne (TFUE) ${ }^{12}$. Leur reconnaître des droits subjectifs constituerait selon certains juristes une avancée considérable. Pourtant, en l'état actuel de notre droit, seules les personnes physiques et morales sont des sujets de droit. Or, comme le souligne Marie-Angèle Hermitte, «si le régime juridique distinct des personnes physiques et morales est une simple construction juridique comme on le pense le plus souvent aujourd'hui, une institution résultant d'actes de volonté du souverain et non pas le résultat d'une nature humaine, alors on peut reconnaître aux non-humains la qualité de sujets de droit, même si leurs droits peuvent être profondément différents des nôtres ${ }^{13}$ ».

Des juristes de plus en plus nombreux prêchent ainsi en faveur de la reconnaissance aux animaux de droits subjectifs qui leur seraient propres et qui pourraient être défendus dans le cadre d'actions en justice. Ce nouvel «habit juridique», qu'ils nomment "personnalité animale ${ }^{14} »$, est devenu leur credo, défendu avec une foi inébranlable et une certaine force de persuasion. Faut-il élever juridiquement l'animal au rang de personne pour en faire un sujet de droits? Est-ce faisable sur un terrain strictement juridique? Nous nous pencherons tout d'abord sur les raisons qui animent ces juristes partis en croisade pour les sans-droits que sont les animaux (1) avant d'aborder

12. L'article 13 TFUE prévoit que «Lorsqu'ils formulent et mettent en œuvre la politique de l'Union dans les domaines de l'agriculture, de la pêche, des transports, du marché intérieur, de la recherche et développement technologique et de l'espace, l'Union et les États membres tiennent pleinement compte des exigences du bien-être des animaux en tant qu'êtres sensibles, tout en respectant les dispositions législatives ou administratives et les usages des États membres en matière notamment de rites religieux, de traditions culturelles et de patrimoines régionaux».

13. M.-A. Hermitte, «La nature, sujet de droit?», Annales. Histoire, Sciences Sociales, $\mathrm{n}^{\circ} 1$, 2011, p. 173-212.

14. J.-P. Marguénaud, F. Burgat et J. Leroy, «La personnalité animale», art. cit. V. aussi, C. Regad, C. Riot et S. Schmitt (dir.), La personnalité juridique de l'animal. 1. L'animal de compagnie, Paris, LexisNexis, 2018. 
les conséquences provoquées par la promotion demain de cette personnalité animale (2).

\section{LES RAISONS DE LA PROMOTION DE L'ANIMAL AU RANG DE «PERSONNE » JURIDIQUE}

Quelle est la situation juridique actuelle de l'animal? (1.1) Quelle situation plus satisfaisante ces juristes proposent-ils d'envisager à l'avenir? (1.2)

\subsection{LA SITUATION ACTUELLE : L'EXTRACTION INCOMPLÈTE DE LA CATÉGORIE DES CHOSES OU DES BIENS}

Le juriste raisonne à partir d'une classification en catégories juridiques héritée de Gaius qui distinguait initialement entre les personnes, les choses et les actions. Cette dernière catégorie ayant disparu, on reste aujourd'hui face à une distinction binaire qui fait que d'un côté existe l'être (soit la personne) et de l'autre l'avoir (soit les choses). Où se situe alors l'animal dans cette summa divisio personnes-choses? Là où d'autres marchent sur l'eau, l'animal serait en «lévitation» au-dessus des catégories juridiques, pour reprendre la très belle expression de Jean-Pierre Marguénaud ${ }^{15}$.

L'animal doit-il être rangé parmi les biens? Si l'animal bénéficie d'un statut spécial suite à la reconnaissance générale de son caractère d'être vivant et sensible dans le Code civil ayant permis de l'extraire de cette catégorie, le régime des biens lui reste toutefois appliqué par défaut comme le précise l'article 515-14 du Code civil («sous réserve des lois qui le protègent»). Il est d'ailleurs indéniable qu'il demeure un objet de propriété ${ }^{16}$. L'animal doit-il figurer parmi les choses? Grégoire Loiseau plaidait dès 2006 pour l'édification

15. J.-P. MARguÉnAud, «La personnalité juridique des animaux», D. 1998, p. 209. Pour cet auteur, ce qui atteste d'autant plus de cette lévitation depuis la réforme de 2015, ce sont les «versions modifiées des articles 522, 524, 528, 533, 564 et 2501 destinées à [...] éradiquer toutes les tournures directes ou indirectes affirmant ou laissant sous-entendre que les animaux sont des meubles, des immeubles ou des objets»: J.-P. MARGUÉnAud, «L'entrée en vigueur de "l'amendement Glavany" : un grand pas de plus vers la personnalité juridique des animaux», RSDA, n 2, 2014, p. 15-44, spéc. p. 22.

16. V. à ce sujet la très belle thèse de L. BoIsSEAu-SOWInSKI, La désappropriation de l'animal, Limoges, PULIM, 2013, qui démontre que «l'application du droit de propriété sur l'animal est non seulement une source d'incohérences juridiques mais encore un frein à sa protection. Cela justifie qu'il convienne d'opérer la "désappropriation" de l'animal, en abolissant l'application du droit de propriété sur celui-ci». 
d'un «droit des choses ${ }^{17} »$ au voisinage du droit des biens, choses qui ne relèveraient pas des «objets de désir» mais vues comme des « objets de respect». Il proposait dès lors pour ces choses, dont l'animal faisait partie, un statut ad hoc prenant en compte leur spécificité et permettant de les protéger du droit des biens. Même s'il n'a jamais abandonné cette idée, Grégoire Loiseau reconnaîtra tout de même que la réforme du Code civil de 2015 a désormais permis à «l'être» de prendre le pas sur l'«avoir ${ }^{18}$... L'animal appartient-il alors aux centres d'intérêts? Cette idée de créer une catégorie sui generis, insérée de façon complémentaire entre les personnes et les choses, a été défendue par Gérard Farjat ${ }^{19}$. Il proposait d'y rattacher les animaux au même titre qu'un très grand nombre d'autres «entités fortes et familières de notre vie»: la famille, le groupe de sociétés, l'entreprise, l'embryon, le cadavre, la nature... Sorte de succédané à la personnalité juridique, les «centres d'intérêts » étaient résolument conçus comme une notion de fait, à géométrie juridique variable, permettant une personnalisation juridique des intérêts en jeu tout en étant dépourvue toutefois du droit d'agir, ce qui distinguait ainsi très nettement cette notion de celle de "personne juridique». Cette catégorie avait ainsi pour ambition de faire bénéficier l'animal d'une protection particulière et renforcée dépassant celle des choses mais n'atteignant pas celle des personnes. La proposition faite par certains juristes de consacrer une personnalité animale va ainsi bien au-delà.

\subsection{LA SITUATION EN DEVENIR : UNE NÉCESSAIRE PROGRESSION PAR LA RECONNAISSANCE D'UNE PERSONNALITÉ ANIMALE}

Force est de constater que dans certains systèmes étrangers de common law, des actions en justice destinées à faire reconnaître l'animal comme une personne ont été couronnées de plus ou moins de succès ${ }^{20}$. Ainsi, si certains philosophes comme Paola Cavalieri et Peter Singer ont plaidé en faveur de

17. G. Loiseau, «Pour un droit des choses», D. 2006, p. 3015.

18. G. LoISEAU, «L'animal et le droit des biens», RSDA, nº 1, 2015, p. 423.

19. G. FARJAT, «Entre les personnes et les choses, les centres d'intérêts - Prolégomènes pour une recherche», RTD civ. 2002, p. 221.

20. C'est principalement à l'étranger qu'ont émergé des litiges stratégiques visant à obtenir par la voie juridictionnelle la reconnaissance d'une personnalité juridique non-humaine et notamment le droit de certains animaux appartenant à des espèces sauvages à ne pas vivre enfermés. Aux États-Unis, ces litiges ont notamment été portés par le Non-human Rights Project (NhRP), association créée par l'avocat Steven Wise: S. WiSE, Tant qu'il $y$ aura des cages. Vers les droits fondamentaux des animaux, Villeneuve d'Ascq, Presses universitaires du Septentrion, 2016. 
l'extension des droits de l'homme aux grands singes ${ }^{21}$, le tribunal argentin de Mendoza, par un jugement en date du 3 novembre 2016, a reconnu une femelle chimpanzé comme «personne non humaine» pour la libérer de sa cage du zoo de Mendoza et la faire transférer dans un sanctuaire pour chimpanzés ${ }^{22}$. Faisant expressément référence à l'article $4^{23}$ d'une Déclaration universelle des droits de l'animal (DUDA) pourtant dépourvue de toute force obligatoire, le juge Maria Alejandra Mauricio a de façon remarquable considéré que:

«les animaux doivent avoir des droits fondamentaux et relever d'une législation en concordance avec ces droits pour les protéger dans la situation particulière où ils se trouvent et en fonction du degré d'évolution que la science a déterminé qu'il puisse atteindre. Il ne s'agit pas de leur accorder les mêmes droits qu'aux êtres humains; il s'agit d'accepter et de comprendre une fois pour toutes qu'ils sont des êtres sensibles ayant une personnalité juridique avec des droits fondamentaux parmi lesquels ceux de naître, vivre, se développer et mourir dans un environnement adapté à leur espèce ${ }^{24} »$.

Cette expérience étrangère peut-elle inspirer nos juges? En l'état actuel de notre droit, rien ne s'oppose conceptuellement à ce que l'animal puisse bénéficier de la qualité de personne juridique, celle-ci n'étant en rien réductible à la personne humaine. Déjà en 1909, le juriste René Demogue affirmait que «la qualité de sujet de droit appartient aux intérêts que les hommes vivant en société reconnaissent comme suffisamment importants pour les protéger par le procédé de la personnalité juridique ${ }^{25} »$. Ainsi, selon cet éminent juriste, «il s'agit de poser une règle technique: est-il commode pour centraliser des résultats souhaitables de considérer même des animaux comme

21. P. Cavalieri et P. Singer, "Tous les animaux sont égaux: le projet "Grands singes" », Mouvements, $\mathrm{n}^{\circ}$ 45-46, 2006/3, p. 22-35.

22. J.-P. MARGUÉNAUD, «La femelle chimpanzé Cécilia, premier animal reconnu comme personne juridique non humaine», RSDA, n 2, 2016, p. 15.

23. Art. 4 de la DUDA, proclamée solennellement le 15 octobre 1978, dans sa version révisée en 1989: «1. L'animal sauvage a le droit de vivre libre dans son milieu naturel et de s'y reproduire. 2. La privation prolongée de sa liberté, la chasse et la pêche de loisir, ainsi que toute utilisation de l'animal sauvage à d'autres fins que vitales, sont contraires à ce droit». Il est à noter qu'en 2018 une version totalement remaniée a été proposée par la Fondation Droit animal, éthique et sciences (LFDA).

24. V. Y. TARDY, «Cécilia, le premier chimpanzé reconnu personne non humaine dotée de droits fondamentaux. Commentaire en ligne du jugement du tribunal civil de Mendoza (Argentine) du 03 novembre 2016», 10 janv. 2018: https://www.ensemblepourlesanimaux. org/project/cecilia-le-premier-chimpanze-reconnu-personne-non-humaine-dotee-de-droitsfondamentaux/ [consulté le 6 juill. 2021].

25. R. Demogue, «La notion de sujet de droit», RTD civ. 1909, p. 611. 
des sujets de droits ${ }^{26}$ ? ». Si certains défendent l'idée que l'animal serait déjà un sujet de droit naissant ${ }^{27}$, un «analogon de la personnalité ${ }^{28}$ », d'autres rappellent que la personnalité morale est une création prétorienne, ce qui plaide en faveur d'une reconnaissance idoine de la «personnalité animale ${ }^{29}$ ». Jean-Pierre Marguénaud souligne que c'est dans sa célèbre jurisprudence dite Comité d'établissement de Saint-Chamond ${ }^{30}$ que la $2^{\mathrm{e}}$ chambre civile de la Cour de cassation a consacré la théorie de la réalité technique élaborée par Léon Michoud en affirmant que «la personnalité civile n'est pas une création de la loi; qu'elle appartient, en principe, à tout groupement pourvu d'une possibilité d'expression collective pour la défense d'intérêts licites, dignes, par suite, d'être juridiquement reconnus et protégés ${ }^{31}$ ", position confirmée deux années plus tard lorsque sa chambre commerciale ${ }^{32}$ reconnaîtra également la personnalité à la masse des obligataires bien qu'aucun texte n'y ait fait une quelconque allusion! L'animal aurait déjà des droits au même titre que les personnes morales ou la masse des créanciers puisqu'il est indéniable qu'il dispose d'un intérêt propre, distinct de celui de son maître lorsqu'il est approprié, intérêt qui est susceptible d'être défendu et mis en œuvre par des organes tels que les associations de protection animale. À noter d'ailleurs que ces droits n'impliqueraient pas forcément des devoirs, à l'instar d'une masse des créanciers titulaire de droits et non de devoirs.

Il ne serait donc pas nécessaire d'en passer par une nouvelle réforme législative mais de réussir à convaincre le juge dans un contentieux particulier de promouvoir l'animal au rang de «personne juridique» pour lui assurer au cas par cas une meilleure protection ${ }^{33}$.

26. Ibid., p. 637, comme le rappelle J.-P. MARGUÉNAUd, « Actualité et actualisation des propositions de René Demogue sur la personnalité juridique des animaux», RJE, n 1, 2015, p. 75.

27. V. les propos de A. Couret, dans sa note sous l'arrêt Cass. $1^{\text {re }}$ civ., 8 oct. 1980: D. 1981, p. 361.

28. E. Engelhardt, De l'animalité et de son droit, Paris, A. Chevalier-Marescq, 1900, p. 124.

29. V. en ce sens R. Nerson, «La condition de l'animal au regard du droit», D. 1963, chron. p. 1, qui affirme que «sur le plan technique, rien ne fait sérieusement obstacle à une organisation juridique où l'animal serait directement traité en sujet de certains droits»; C. DaiguePerse, «L'animal, sujet de droit: réalité de demain», Gaz. Pal. 1981, doct., p. 160 ; J.-P. Marguénaud, L'animal en droit privé, Limoges, PULIM, 1993, spéc. p. 307-406; J.-P. MARguÉnAUd, F. BuRgat et J. Leroy, « La personnalité animale», art. cit.

30. Cass. $2^{\mathrm{e}}$ civ., 28 janv. 1954, $\mathrm{n}^{\mathrm{o}}$ 54-07.081: Bull. civ II, $\mathrm{n}^{\circ} 32$.

31. V. par ex. J.-P. MARguÉnAud, «Actualité et actualisation des propositions de René Demogue sur la personnalité juridique des animaux», art. cit., spéc. p. 76.

32. Cass. com., 17 janv. 1956: D. 1956, p. 256, note R. Houin.

33. On observe de façon embryonnaire en France ces contentieux stratégiques portés par des associations de protection animale: S. NADAUD, «Le contentieux stratégique en droit animalier. L'exemple des litiges stratégiques visant à faire interdire la détention et l'utilisation 


\section{LES CONSÉQUENCES DE CETTE PROMOTION DE L'ANIMAL AU RANG DE "PERSONNE » JURIDIQUE}

Si on reconnaissait à l'animal la personnalité juridique technique, peut-on affirmer de façon péremptoire que pour l'animal cela changerait tout (2.1) et que pour l'homme cela ne changerait rien (2.2)?

\subsection{POUR L'ANIMAL : CELA CHANGERAIT TOUT !}

Deux principaux arguments sont régulièrement soutenus pour écarter la personnalité animale: c'est compliqué et ça ne sert pas à grand-chose! Pour remettre en cause la faisabilité d'une telle innovation juridique, certains mettent en avant les nombreux intérêts liés à l'exploitation ou l'utilisation des animaux (économiques, sanitaires, scientifiques, culturels, religieux, de loisir, etc.) comme autant d'obstacles insurmontables à la reconnaissance de cette personnalité animale, là où d'autres veulent bien admettre que la personnalité animale ne reviendrait finalement qu'à poser une simple règle technique mais font valoir du peu d'utilité de la personnalité animale. Ainsi, dès 1990, Anne-Marie Sohm-Bourgeois ${ }^{34}$ va par exemple considérer qu'il ne s'agit en somme que d'une personnification à effets limités, ne s'appliquant qu'à certains animaux à l'exclusion de beaucoup d'autres et ne permettant d'attribuer à ces bénéficiaires que des droits patrimoniaux et extrapatrimoniaux extrêmement sommaires, concluant ainsi: "n'est-ce pas plus raisonnable d'améliorer la condition des animaux sans en changer la nature juridique?». En ce sens, d'autres auteurs dont les thèses font référence en droit animalier ${ }^{35}$ considèrent également que l'amélioration de la condition des animaux pourrait parfaitement être menée à bien par d'autres techniques ou instruments. À tous ces arguments, René Demogue répondait de façon prémonitoire en 1909 ceci: «l'extension de la qualité de sujet de droit pourrait tout aussi bien peut-être être remplacée par d'autres procédés techniques. Car différents instruments peuvent fort bien amener au même résultat [...] Mais on aurait au fond le procédé technique de la

des animaux sauvages dans les cirques», in C. Boyer-Capelle et E. Chevalier (dir.), Contentieux stratégiques. Approches sectorielles, vol. 1, Paris, LexisNexis, à paraître fin 2021.

34. A.-M. SOHM-Bourgeois, «La personnification de l'animal: une tentation à repousser», D. 1990 , p. 33.

35. S. Desmoulin-Canselier, L'animal, entre Science et Droit, Aix-en-Provence, Presses universitaires d'Aix-Marseille, 2006; P.-J. Delage, La condition animale: essai juridique sur les justes places de l'Homme et de l'animal, Paris, Mare \& Martin, 2016. 
personnification sans le nom. Et ne vaut-il pas mieux de deux constructions possibles choisir la plus simple ${ }^{36}$ ?».

Attribuer la personnalité juridique technique aux animaux pour préserver leurs droits les plus primordiaux (droit à des aliments, à des soins, à ne pas subir de souffrances inutiles, etc.), protégeant ainsi leur intégrité, n'est pourtant ni déraisonnable, ni inutile, ni complètement illusoire. Même si la personnalité animale ne semble pas de prime abord révolutionner la condition animale, elle permettra de continuer à la faire progresser, par la réalisation d'avancées particulièrement attendues mais que le droit ne peut pour l'heure résoudre avec les outils actuels ${ }^{37}$. Le seul argument qui pourrait être sujet à discussion est selon nous celui qui porte sur l'exclusion de certains animaux de cette avancée juridique. La personnalité animale semble en effet être principalement conçue pour les animaux proches de l'homme. En écarter les animaux sauvages serait toutefois une ségrégation scientifique et juridique peu acceptable pour ces êtres vivants méritant eux aussi de bénéficier de cette personnalité technique tant à titre individuel qu'au titre de leur appartenance à une espèce ${ }^{38}$. La personnalité animale devrait en effet permettre pour tous les animaux de défendre des intérêts tant individuels que collectifs, même si cela suppose de consacrer des droits différents aux animaux en fonction de leur situation, sans entamer en rien l'égalité animale puisqu'à situation différente, traitement différent.

Si la personnalité animale fera progresser la protection des animaux en leur accordant certains droits à un moment donné, doit-on considérer qu'elle aura des répercussions néfastes sur les droits de l'homme?

\subsection{POUR L'HOMME : CELA NE CHANGERAIT RIEN?}

«À force de vouloir considérer les animaux comme des personnes, on finit forcément par considérer les personnes comme des animaux» écrivait Francis Wolff ${ }^{39}$. Pour paraphraser l'ouvrage de Jean-Louis Harouel, Les droits de l'homme contre le peuple ${ }^{40}$, faut-il croire qu'attribuer des droits

36. R. Demogue, art. cit.

37. Tel est par exemple le cas de la réparation de la souffrance subie par un animal sur le plan civil.

38. V. en ce sens J.-P. MARguÉnAud, «Actualité et actualisation des propositions de René Demogue sur la personnalité juridique des animaux», art. cit., spéc. p. 80-83.

39. Cité par Th. Lepeltier, L'imposture intellectuelle des carnivores, Paris, M. Milo, 2017.

40. J.-L. Harouel, Les droits de l'homme contre le peuple, Paris, Desclée de Brouwer, 2016. 
aux animaux se fera nécessairement contre l'homme et contre les droits de l'homme ${ }^{41}$ ?

La personnalité animale telle que défendue n'est pas une personnalité anthropomorphique. En effet, «conférer aux animaux une personnalité juridique à taille humaine serait pour eux une promotion; mais ce serait une promotion inadaptée parce qu'elle leur conférerait des droits inutiles et les exposerait à des obligations grotesques ${ }^{42}{ }$. Il s'agit au contraire d'attribuer une personnalité singulière, différente de celle donnée aux personnes physiques et morales. Il n'y a donc aucun risque d'amalgame, aucun risque que cette personnalité animale vienne élever l'animal au rang égal de l'humain, ni abaisser l'humain au rang de l'animal. Attribuer des droits à l'animal ne conduira pas à minorer les droits de l'homme mais plutôt à les élever: comme le disait Kant, « les devoirs que nous avons en fait envers les animaux sont des devoirs envers l'humanité». Il s'agit ni plus ni moins que d'élever l'homme vers une humanité qui intègre les droits des animaux, qui prenne en compte la condition animale bien plus qu'elle ne le fait aujourd'hui. L'étude de la jurisprudence de la Cour européenne des droits de l'homme démontre à l'envi que ces dernières années les droits de l'homme se sont enrichis «de la pleine conscience de l'humanité sur le devenir des autres espèces, des écosystèmes naturels, et plus largement de l'environnement ${ }^{43}$ ». Tenant compte des préoccupations croissantes en ce domaine, la Cour a ainsi considéré d'une part que la protection de l'environnement, qui est d'intérêt général, peut constituer un but légitime pouvant justifier certaines restrictions apportées par les autorités publiques à des droits garantis, et d'autre part, qu'une atteinte portée à l'environnement peut sous certaines conditions être constitutive d'une violation d'un droit conventionnellement protégé.

La Cour suit une trajectoire jurisprudentielle relativement similaire s'agissant de la protection des animaux. Par exemple, dans une affaire relative au refus d'octroyer un permis autorisant l'abattage rituel en accord avec les pratiques strictes d'une association juive orthodoxe ${ }^{44}$, la Cour européenne des

41. S. NADAUD, «Droits de l'homme et droits des animaux: la quadrature du cercle?», RTDH 2021, p. 373.

42. . J.-P. MARguÉnAUd, «La personnalité juridique des animaux», D. 1998, p. 207.

43. Opinion partiellement concordante et partiellement dissidente du juge Pinto De Albuquerque sous CEDH, Gde ch., 26 juin 2012, n 9300/07, Herrmann c. Allemagne.

44. CEDH, Gde ch., 27 juin 2000, $\mathrm{n}^{\circ}$ 27417/95, Cha'are Shalom Ve Tsedek c. France. De l'avis de la Cour, il n'y aurait ingérence dans la liberté de manifester sa religion que si l'interdiction de pratiquer légalement cet abattage conduisait à l'impossibilité pour les croyants ultra-orthodoxes de manger de la viande provenant d'animaux abattus selon les prescriptions requises par leur religion. La Cour estime que le droit à la liberté religieuse 
droits de l'homme va écarter toute ingérence dans le droit de la requérante à la liberté de manifester sa religion, qui reste préservée par la possibilité de se procurer et de manger une viande conforme à ses préceptes religieux. Il est à noter que sur cette épineuse question de l'abattage rituel, la Cour de justice de l'Union européenne s'est pour sa part récemment prononcée sur la compatibilité avec l'article $10 \S 1$ de la Charte des droits fondamentaux (qui garantit le droit de manifester sa religion) d'une réglementation nationale imposant un procédé d'étourdissement réversible et insusceptible d'entraîner la mort de l'animal. S'appuyant sur une lecture des dispositions de la Charte à la lumière des conditions actuelles et du contexte sociétal, la Cour de Luxembourg estime que les mesures prises dans le cadre du décret belge en cause permettent d'assurer un juste équilibre entre l'importance attachée au bien-être animal et la liberté de manifester leur religion des croyants juifs et musulmans et, par conséquent, sont proportionnées ${ }^{45}$.

Il n'y aura donc point d'antagonisme entre les droits des animaux et les droits de l'homme, car les premiers n'auront pas vocation à remettre en cause les seconds mais plutôt à les faire évoluer encore davantage pour prendre en considération la dignité animale, chaque fois que cela ne heurte pas le respect de la dignité humaine, chaque fois finalement que dignité animale et dignité humaine se conforteront mutuellement ${ }^{46}$. La personnalité animale constitue sans aucun doute l'instrument à privilégier à l'avenir pour que ce qui semble être une utopie aujourd'hui devienne demain une réalité.

garanti par l'article 9 de la Convention ne saurait aller jusqu'à englober le droit de procéder personnellement à l'abattage rituel et à la certification qui en découle, dès lors que, comme il a été dit, la requérante et ses membres ne sont pas privés concrètement de la possibilité de se procurer et de manger une viande jugée par eux plus conforme aux prescriptions religieuses.

45. CJUE, Gde ch., 17 déc. 2020, C-336/19, Centraal Israëlitisch Consistorie van België e.a., et a. La Cour souligne ici que «le bien-être animal, en tant que valeur à laquelle les sociétés démocratiques contemporaines attachent une importance accrue depuis un certain nombre d'années, peut, au regard de l'évolution de la société, être davantage pris en compte dans le cadre de l'abattage rituel et contribuer ainsi à justifier le caractère proportionné d'une réglementation telle que celle en cause au principal» (§ 77). Ainsi, «dans un contexte en évolution sur les plans tant sociétal que normatif [...], le législateur flamand a pu adopter, à l'issue d'un vaste débat organisé à l'échelle de la Région flamande, le décret en cause au principal, sans excéder la marge d'appréciation que le droit de l'Union confère aux États membres quant à la conciliation nécessaire entre l'article 10, paragraphe 1, de la Charte et l'article 13 TFUE» (§ 79).

46. J.-M. Coulon, «Antagonisme ou complémentarité entre les droits humains et les droits des animaux», RSDA, $\mathrm{n}^{\circ} 1,2010$, p. 287. 